Relations industrielles

Industrial Relations

\title{
Réalisations professionnelles
}

\section{La Commission d'apprentissage de l'industrie de la chaussure}

\section{Léonce Girard}

Volume 5, numéro 8, mai 1950

URI : https://id.erudit.org/iderudit/1023366ar

DOI : https://doi.org/10.7202/1023366ar

Aller au sommaire du numéro

Éditeur(s)

Département des relations industrielles de l’Université Laval

ISSN

0034-379X (imprimé)

1703-8138 (numérique)

Découvrir la revue

Citer cet article

Girard, L. (1950). Réalisations professionnelles : la Commission d'apprentissage de l'industrie de la chaussure. Relations industrielles / Industrial Relations, 5(8), 73-74. https://doi.org/10.7202/1023366ar

Tous droits réservés @ C Département des relations industrielles de l’Université Laval, 1950
Ce document est protégé par la loi sur le droit d'auteur. L'utilisation des services d'Érudit (y compris la reproduction) est assujettie à sa politique d'utilisation que vous pouvez consulter en ligne.

https://apropos.erudit.org/fr/usagers/politique-dutilisation/ 
tre la vieillesse; de plus, les risques étant répartis sur tous les employés, le coût est beaucoup moindre pour l'employeur.

Le paiement d'une pension apparaît comme un supplément de salaire que l'employeur, en saine philosophie sociale, serait tenu de verser à son employé pour lui permettre de faire des épargnes en prévision de sa vieillesse, et que l'employé au- rait droit et serait justifiable de réclamer.

Le droit à une pension fait donc partie de la rémunération du travailleur, et la cotisation que l'employeur est tenu d'y verser est proprement une partie du salaire de l'employé. Il suit que le refus d'un employeur d'établir un fonds de pension équivaut à un refus d'accorder une augmentation de salaire.

\section{RÉALISATIONS PROFESSIONNELLES}

\section{La Commission d'apprentissage de l'industrie de la chaussure \\ Léonce Girard}

$\mathrm{Si}$, un jour ou l'autre, vous vous êtes intéressé à une commission d'apprentissage, on vous a sans doute posé cette question très à la mode: «Qu'estce que les commissions d'apprentissage sont venues faire dans l'industrie ou dans l'enseignement?»

De façon générale, la réponse est très simple: elles ont voulu promouvoir la formation professionnelle en faisant valoir le point de vue de l'industrie.

\section{Vue d'ensemble}

Mais, pour répondre de façon plus explicite à cette question, je prendrai, comme exemple, la Commission d'apprentissage de l'industrie de la chaussure, que je connais davantage, et je m'efforcerai d'exposer les moyens qu'elle a employés pour promouvoir l'enseignement en l'adaptant constamment aux besoins actuels de l'industrie.

A cette fin, elle a tout d'abord dressé, par une statistique soignée, le bilan de la main-d'oeuvre de l'industrie et a déterminé, de façon précise, le nombre de ses apprentis et de ses compagnons de façon à ne pas l'encombrer tout en lui assurant des recrues qualifiées en quantité suffisante.

Elle a tenu, en second lieu, à ce que les écoles enseignent le fonctionnement des machines employées de nos jours dans l'industrie et a recommandé la mise au rancart de tout l'outillage démodé ou sans utilité. De même, elle a rédigé la liste des opérations importantes qui doivent être enseignées pour que l'élève devienne un ouvrier qualifié et non seulement un manoeuvre ou même un ouvrier spécialisé.
La commission d'apprentissage a tenu à faire elle-même le choix des professeurs de techniques et a suggéré, à la direction des écoles, ceux qui lui paraissaient les plus aptes à bien enseigner le métier tel qu'il se pratique actuellement; elle a aussi tenu la direction et les professeurs des écoles au courant de tous les procédés nouveaux, de tous les développements de la technique, de façon que l'école soit à date dans son enseignement, et elle a recommandé l'abandon de tout enseignement relatif à des opérations présentement disparues dans l'industrie. Elle a insisté pour que les cours théoriques (les langues, les mathématiques, le dessin, la physique, l'hygiène, l'anatomie) soient enseignés, non pas de façon purement théorique, mais en regard des besoins de l'industrie.

Avec l'aide des ministères du Travail, du Bien-être social et de la jeunesse, elle a fondé deux écoles de chaussure, une à Montréal, l'autre à Québec; elle a donné, dans ces écoles, des cours du jour et du soir; elle a organisé des cours d'usines à travers la province pour les employés qui ne peuvent pas se rendre à ces écoles; enfin, elle a publié un service de renseignements pour tous les employeurs et employés de l'industrie que les cours n'atteignaient pas.

Signalons encore que la commission d'apprentissage a fait en sorte que les élèves qui ont acquis une compétence suffisante aux cours du jour, à l'école, obtiennent et conservent une position stable dans l'industrie. Ce service profite surtout aux élèves du jour, vu que la plus grande majorité des apprentis qui suivent les cours de perfectionnement le soir, à l'école ou dans les usines, ont déjà un emploi dans l'industrie.

Voilà, dans son ensemble, l'attitude de la commission d'apprentissage de l'industrie de la chaus- 
sure. D'autres commissions d'apprentissage ont adopté des procédés différents, d'après les besoins de leur industrie respective, et ont accompli un travail non moins considérable.

\section{Quelques résultats}

Mais, avant de faire une étude détaillée des méthodes et des procédés employés par la commission d'apprentissage de la chaussure, une question préalable se pose: Cette commission d'apprentissage, après quatre ans d'existence, a-t-elle obtenu des résultats appréciables, dignes d'être portés à l'attention du public?

Voyons plutôt les faits. Il y a deux ou trois ans, des jeunes gens de quinze à vingt ans étaient sans argent, sans orientation dans la vie, sans emploi; aujourd'hui, après avoir fait des études sous les auspices de la commission d'apprentissage de la chaussure, ils sont des hommes-clefs dans cette industrie: des gérants, des assistants-gérants, des ouvriers qualifiés dans des fabriques importantes. On admettra sans doute qu'il vaut la peine de chercher les causes d'un tel résultat.

Depuis trois ans, 31 élèves ont complété leurs cours du jour à l'école de Montréal. La commission d'apprentissage est en contact avec 29 d'entre eux. Aujourd'hui, voici ce que sont devenus ces 29 apprentis:

1 est gérant, 2 sont assistants-gérants, 1 est contremaître, 4 sont assistants-contremaîtres, 1 est patroniste, 2 sont modeleurs, 3 sont vendeurs, 4 sont ouvriers qualifiés, 3 sont hommes-clefs pour surveiller la fabrication générale, 4 sont ouvriers spécialisés, 1 est cordonnier à son compte, 1 est cordonnier pour l'armée, 1 est étudiant à l'université, 1 est religieux.

\section{Le placement}

La formation professionnelle a pour but de préparer les salariés à des emplois rémunérateurs dans l'industrie et le commerce. $\mathrm{Si}$, une fois formé, l'élève ne réussit pas à se procurer un emploi stable, toute la raison d'être de la formation professionnelle se trouve, par le fait même, manquée. A quoi bon, en effet, spécialiser ou qualifier un employé, s'il y a ensuite impossibilité de le placer?

Il n'est donc pas surprenant que la commission d'apprentissage ait accordé une attention spé- ciale au placement des élèves de l'école qui ont acquis un certain degré de compétence.

A cette fin, la commission d'apprentissage prend soigneusement note des qualifications des apprentis en vue d'en faire une recommandation exacte aux manufacturiers; au besoin, une lettre est adressée à toutes les firmes de la région, donnant le nom des élèves qui ont complété leur stage à l'école et indiquant les opérations importantes qu'ils sont en mesure de remplir, au moins à titre d'apprentis.

La commission d'apprentissage s'est aussi préoccupée de faire appel aux sentiments chrétiens et au sens social des patrons; elle a demandé à nombre d'employeurs de considérer les apprentis qui sortent de l'école, non pas comme des étrangers, mais comme leurs propres enfants et de tâcher de leur découvrir des qualités, avec la même bienveillance que s'il s'agissait de leurs fils ou filles. Les dirigeants de l'industrie comprennent qu'il est de beaucoup préférable de prendre à leur service un nouvel employé qui sort de l'école avec une formation sociale chrétienne, plutôt que des inconnus dont les principes et les convictions ne sont pas toujours recommandables.

Enfin, la commission d'apprentissage a demandé aux industriels de l'aviser avant de congédier un élève à leur emploi. Cette demande a été agréée de tous. La commission ne demande jamais à un employeur de garder un élève, pour quelque raison que ce soit, mais de l'aviser au cas où il voudrait le renvoyer pour qu'elle ait la possibilité de lui trouver un autre emploi. Et pourquoi ? Pour que l'élève ne soit pas découragé, ne perde pas son amour du métier et son enthousiasme à apprendre et à progresser.

Actuellement, comme on l'a vu plus haut, les anciens élèves de l'école qui travaillent dans l'industrie ont des positions stables.

Un fait important à noter. Il y a eu du chômage saisonnier en décembre 1948, en janvier 1949, en décembre 1949 et en janvier 1950. Pourtant, pas un des élèves de l'école n'a été congédié durant ces périodes. C'est le signe que, malgré les déficiences propres à leur âge, ils sont des ouvriers qualifiés. Comment la commission d'apprentissage a-t-elle réussi à en faire des employés qualifiés ? C'est ce que l'on verra dans un prochain article. 